\title{
Victor Michel Jean-Marie Thébault zum 125. Geburtstag am 6. März 2007
}

\author{
Evgeniy D. Kulanin und Oleh Faynshteyn \\ Evgeniy D. Kulanin promovierte 1986 im Fach Mathematik an der Moskauer Univer- \\ sität. Zur Zeit arbeitet er als Professor der Fakultät der informatischen Technologien \\ der Moskauer städtischen psychologisch-pädagogischen Universität. Er ist Autor rus- \\ sischsprachiger Lehrbücher und Aufgabensammlungen in der Elementarmathematik. \\ Sein Hauptinteresse gilt der Elementargeometrie, speziell der Dreiecksgeometrie. \\ Oleh Faynshteyn schloss 1970 sein Studium an der Technologischen Hochschule \\ M.W. Lomonossow in Odessa (Ukraine) ab und war danach als Ingenieur tätig. Später \\ studierte er Mathematik an der Staatlichen Gorki-Universität in Charkov. Nach dem \\ Abschluss dieses Studiums wurde er als Mathematiklehrer tätig.
}

\section{Einleitung}

Am 6. März 2007 wurde der berühmte französische Mathematiker Victor Thébault 125 Jahre alt. Er ist in der ganzen Welt für sein geometrisches Schaffen bekannt, insbesondere für die Lösung von zahlreichen und sehr interessanten Aufgaben auf dem Gebiet der Geometrie und Zahlentheorie.

\section{Biographie}

V. Thébault wurde am 6. März 1882 in Ambrières-le-Grand (Mayenne), Frankreich, geboren. Sein Vater war Weber [3]. Der Lehrer der lokalen Grundschule erkannte die ungewöhnlichen mathematischen Fähigkeiten des jungen Thébault und verschaffte ihm ein Sti-

Dieser Artikel ist dem 125. Geburtstag des berühmten französischen Elementargeometers V. Thébault gewidmet. Er beinhaltet eine kurze Biographie und ergründet die Prinzipien seines wissenschaftlich-pädagogischen Schaffens; dabei gehen die Autoren auch auf die historischen Sichtweisen der Dreiecksgeometrie ein. Aus der fast unüberblickbaren Anzahl an Aufgaben, die Thébault seiner Nachwelt hinterließ, wurden lediglich einige wenige ausgewählt, wobei nur auf die Lösung seiner wohl berühmtesten Aufgabe genauer eingegangen wird - die drei Umkreise mit kollinearen Mittelpunkten. Es werden schließlich einige weitere Aufgaben untersucht, die eng mit den Aufgaben Thébaults zusammenhängen. 
pendium am Lehrerkolleg der Stadt Laval (Mayenne), wo Thébault von 1898 bis 1901 ausgebildet wurde. Nach dem Abschluss seines Studiums im Kolleg arbeitete V. Thébault zwischen 1902 und 1905 als Schullehrer in Pré-en-Pail (Mayenne), und zwar solange, bis er zum Professor der technischen Schule in Ernée (Mayenne) ernannt wurde. Im Jahre 1909, als Ergebnis eines Erfolgs in Wettbewerbsprüfungen, bekam V. Thébault die Chance auf eine Professur am Lehrerkolleg.

Da das bescheidene Einkommen des Professors seine Familie nicht ernähren konnte, welche zu jener Zeit schon sechs Kinder zählte, war V. Thébault gezwungen, sich vom Unterricht loszusagen. Von 1910 bis 1923 arbeitete er als Fabriksuperintendant in Ernée. Später nahm V. Thébault den Posten des Hauptversicherungsinspektors (Chief Insurance Inspector) in Le Mans (Sarthe) an und siedelte nach der Auszahlung seiner Rente im Jahre 1940 nach Tennie (Sarthe) über.

Diese Zeit nutzte er, ungeachtet seiner Arbeitstätigkeit, intensiv und nutzbringend, indem er sich zusätzlich mit Mathematik beschäftigte. Erstaunlich, wie gut ihm dies gelang. Fairerweise sollte an dieser Stelle angemerkt werden, dass in der Geschichte der Mathematik zahlreiche ähnliche Beispiele existieren, sogar denselben Tätigkeitsbereich betreffend nämlich die Versicherungsbranche. Erwähnenswert wären große Mathematiker wie J. Sylvester (1814-1897) und J. Gram (1850-1916), berühmt durch seine Arbeit an der Gramschen Determinante. J. Gram wurde im Jahre 1896 sogar zum Direktor einer Versicherungsgesellschaft und zum Vorsitzenden des dänischen Versicherungsrates ernannt. Nach modernen Vorstellungen war Thébault jedoch kein Profimathematiker, er ging der Mathematik als einer Freizeitbeschäftigung nach, doch dies schmälert seine Verdienste auf diesem Gebiet in keiner Weise. Was er erreicht hatte, wurde auf der ganzen Welt gewürdigt. Deutlich wird dies durch seine Aufnahme in die American Mathematical Society im Jahre 1932.

Im selben Jahr wurde er auf Empfehlung des Akademiemitgliedes M. d'Ocagne zum $O f$ ficier de l'Instruction Publique ernannt. Dazu war die folgende Passage zu lesen: „Ich persönlich verneige mich vor seinem beachtlichen Talent in der Mathematik, welches in den zahlreichen scharfsinnigen Errungenschaften auf dem Gebiet der elementaren Geometrie deutlich wird: die schier unerschöpfliche Quelle seiner Aufgaben, deren Lösung eine besondere Gabe geschickter mathematischer Denkstrukturen erfordert.“

Im Jahre 1935 wurde V. Thébault zum Chevalier de l'Ordre de la Couronne de la Belgique (Kavalier des Ordens der belgischen Krone) für seine Tätigkeit in der Brüsseler Gesellschaft der Wissenschaft und für die Zusammenarbeit mit den Zeitschriften dieser Gesellschaft (Annalen und Mathesis) ernannt. Im Jahre 1943 wurde eine Auszeichnung mit dem Namen Victor Thébaults ins Leben gerufen. Laut Bestimmung wird sie alle zwei Jahre von der Pariser Akademie der Wissenschaften für Forschungsleistungen in der Geometrie oder Zahlentheorie verliehen, wobei sie ausschließlich Lehrern von Mittelschulen oder sogar von Grundschulen überreicht werden soll.

\section{Die wissenschaftliche Tätigkeit}

In seinem Leben stellte Thébault 15 Veröffentlichungen allein für die Pariser Akademie der Wissenschaften vor und schrieb Hunderte von weiteren Artikeln. Noch größere Berühmt- 
heit erlangte er als Autor äußerst origineller Aufgaben, von denen 582 im American Mathematical Monthly abgedruckt wurden. Alles in allem erdachte Thébault über 1000 verschiedene Aufgaben und hatte darum auf diesem Gebiet keinerlei Konkurrenz.

Die wissenschaftlichen Interessen Thébaults erfassten drei Bereiche der Mathematik - die Geometrie des Dreiecks und des Tetraeders sowie die Zahlentheorie. Er begann sich im ersten Jahrzehnt des 20. Jahrhunderts ausführlich mit der Dreiecksgeometrie zu beschäftigen [4], also in einer Zeit, als die erste Entwicklungsperiode dieser Disziplin beendet war und sie daraus als ein umfangreicherer und breitere Spektren umfassender Bereich der Geometrie hervorging, dabei die Aufmerksamkeit vieler Mathematiker auf sich ziehend. V. Thébault hat in praktisch allen Abschnitten der elementaren Geometrie Ergebnisse erzielt: er untersuchte Dreiecke, Vierecke, Polygone, Kegelschnittlinien und Kubiken, die mit dem Dreieck verbunden sind und vieles ähnliches.

Am Anfang seiner Tätigkeit gelang es ihm, den folgenden Satz zu beweisen:

If a transversal through the centre of the circumscribed circle meets the sides of a triangle in the points $B_{1}, B_{2}, B_{3}$, the circles on $\left(A_{i} B_{i}\right)$ as diameters are concurrent on the circumscribed and nine-point circles.

Der Autor J.L. Coolidge, berühmt für sein Buch Das Traktat über den Kreis und die Sphäre (1916), nahm diesen Satz in seine Arbeit A treatise on the circle and the sphere (S. 113, 1916) auf, ungeachtet der Tatsache, dass das entsprechende Kapitel über jenes Thema bereits geschrieben war.

In der Geometrie des Dreiecks hatte V. Thébault seine besonderen Lieblingsthemen, die er mehrfach aufnahm. Ein solches Thema war der Satz von Feuerbach. V. Thébault untersuchte die Feuerbach-Punkte am umfangreichsten in seinem ersten Artikel, der in Nouvelles Annales de Mathématiques (séries 4, vol. 10, 1910, pp. 271-281) veröffentlicht wurde. In der Folge hat er ein Dutzend Artikel zu diesem Thema veröffentlicht, in denen er die zahlreichen Eigenschaften dieser Punkte aufgezeigt hat. 1935 bewies V. Thébault den nächsten interessanten Satz:

In einem Dreieck ABC sind die vier Dreiecke, deren Eckpunkte auf den Feuerbach-Punkten des Dreiecks ABC liegen, den vier Dreiecken, deren Eckpunkte auf den Fußpunkten der entsprechenden Winkelhalbierenden des Dreiecks ABC liegen, ähnlich.

Viele Ergebnisse erzielte er auch auf dem Gebiet der Geometrie des Tetraeders. Die wissenschaftliche Arbeit V. Thébaults wurde während des Zweiten Weltkriegs nicht unterbrochen, obwohl er während dieser Periode nach Spanien emigrieren musste.

\section{Historisches für und gegen die Dreiecksgeometrie}

Hier erlauben wir uns, ein wenig vom Thema abzuweichen, um über die Geometrie des Dreiecks und ihre Stellung in der Geschichte der Mathematik zu sprechen. Anscheinend war V. Thébault einer „,der letzten Mohikaner“ auf diesem Gebiet, der sich darin weltweite 
Anerkennung verdiente. Natürlich galt er als kein großer Mathematiker, aber alle seine Artikel über die Geometrie des Dreiecks und des Tetraeders wurden in den Ausgaben der Akademien der Wissenschaften von Paris und Brüssel gedruckt, was heute auf dem Gebiet der Dreiecksgeometrie kaum möglich wäre.

Gegen Ende des 19. Jahrhunderts wurde für Fragen der Dreiecksgeometrie sogar ein eigenes Forum auf internationalen mathematischen Kongressen eingeräumt. So betrachtete der Begründer der neuen Geometrie des Dreiecks, der Franzose E. Lemoine, in seinem Vortrag auf einem mathematischen Kongress in Lyon jenen Punkt des Dreiecks, für den die Summe der Quadrate der Abstände zu seinen drei Seiten die Kleinste ist. Der berühmte russische Mathematiker P. Tchebychef hat bemerkt, dass dieser Punkt bereits Gauß bekannt war. In diesem Zusammenhang bat ihn E. Lemoine in einem Brief, der auf den 8.12.1885 datiert ist, ihm die entsprechende Auskunft zu erteilen, damit er seine bibliographischen Notizen ergänzen konnte [20]. Dieser Punkt, der 1847 nach E. Grebes benannt wurde, war schon 1847 nicht neu und wurde 1873 zu Ehren E. Lemoines umbenannt.

Der große deutsche Mathematiker Felix Klein hat im zweiten Band seiner Elementarmathematik vom höheren Standpunkt aus (Geometrie) auf folgende Weise die Geometrie des Dreiecks charakterisiert: „Endlich habe ich noch die sog. Dreiecksgeometrie zu nennen, das ist die Lehre von den ,merkwürdigen“ Punkten und Geraden des Dreiecks; die ganz besonders innerhalb der Schulmathematik als selbständige Disziplin ausgebildet worden ist; auch hier werden Sie mir bestätigen, dass dies Gebiet in demselben Maße, wie es im Schulunterricht in den Vordergrund zu treten pflegt, bei weitergehenden Studien zurücktritt. Wir haben ja früher schon erläutert, in welcher Ecke der projektiven Geometrie diese Dreiecksgeometrie einzuordnen ist. Es handelt sich um die Invariantentheorie derjenigen ebenen Figur, welche aus drei beliebigen Punkten und den beiden imaginären Kreispunkten ihrer Ebene gebildet ist, also tatsächlich um etwas durchaus Partikuläres“ [10].

Dieses Urteil von F. Klein bildete natürlich für die Kritiker der Dreiecksgeometrie eine günstige Argumentationsgrundlage. Seitdem versuchen diese unermüdlich die Unwissenschaftlichkeit dieser Disziplin zu beweisen. Sie repräsentiert in ihren Augen eine Fingerübung für Schullehrer, die von der eigenen mathematischen Unvollkommenheit ablenkt. Hier wäre es passend, den Standpunkt V. Thébaults zu erwähnen, der mit ähnlichen Vorwürfen konfrontiert wurde, da er u.a. zwar interessante arithmetische und theoretische Zahlenaufgaben kreierte, die sich allerdings leicht in die Kategorie der Unterhaltung einordnen lassen: „Einige der Mathematiker demonstrieren eine Tendenz, nicht frei von Verachtung, in solchen Aufgaben nur die unbedeutenden Kleinigkeiten zu sehen. Kleinigkeiten, so soll es sein, jedoch handelt es sich um solche, deren Lösung mehr Scharfsinn, Erfindungsgabe und die feine Zahlenkunst erfordert, als die meisten Fragen, deren Bedeutung als höher angesehen wird. Darüber hinaus erfordert das Studium elementarer Fragestellungen größere Mühen, die als wunderbare intellektuelle Übungen bezeichnet werden können und darum größere Aufmerksamkeit verdienen“ [17]. Weiter bemerkt V. Thébault, dass nicht alle großen Meister der Wissenschaft ähnliche Verachtung gegenüber mathematischer Unterhaltung hegten, und zitiert Euler, Jacobi und andere große Mathematiker.

Obwohl die Geometrie des Dreiecks, besonders einige ihrer ausführlicheren Teile, allzu komplex ist, um sie in die Kategorie der mathematischen Unterhaltungen einordnen zu können, ist sie dennoch um einiges leichter zu begreifen als zum Beispiel die letzten Errun- 
genschaften in der algebraischen Geometrie oder der algebraischen Zahlentheorie. Und es liegt nichts Schlechtes darin, dass diese Disziplin den Akteuren erlaubt, die uneigennützige Freude des Schaffens zu fühlen, da man im Gegensatz zur „echten“ Mathematik kaum mit materiellem Gewinn, Ehrungen oder Ruhm rechnen kann.

In diesem Zusammenhang sei einer der ersten Begründer der Dreiecksgeometrie, A.L. Crelle (1780-1855), erwähnt, der das berühmte Journal A.L. Crelle gegründet hat. In seiner Arbeit Über einige Eigenschaften des ebenen geradlinigen Dreiecks rücksichtlich dreier durch die Winkelspitzen gezogenen geraden Linien, die er im Jahre 1816 verfasste, hat er insbesondere die Punkte, die in der Folge den Namen des französischen Mathematikers H. Brocard erhielten (1875), entdeckt. Crelle bemerkt dazu: „Es ist in der Tat bewundernswürdig, dass eine so einfache Figur, wie das Dreieck, so unerschöpflich an Eigenschaften ist. Wie viele unbekannte Eigenschaften anderer Figuren mag es noch geben?“ (Sammlung, v. I, 1821, p. 176).

Abschliessend soll angemerkt werden, dass, unabhängig von der kritischen Einstellung Kleins, im Band III der unter seiner Redaktion herausgebrachten Enzyklopädie der mathematischen Wissenschaften ein Artikel [2] über Neuere Dreiecksgeometrie mit mehr als 100 Seiten veröffentlicht wurde. Darüber hinaus vertrat F. Klein in seinem Buch Höchste Geometrie die Ansicht, dass die elementare Geometrie durchaus eine eigene wunderbare Bedeutung besitzt. Da nähere Erläuterungen dazu den Rahmen dieses Artikels sprengen würden, verweisen wir die interessierten Leser auf den packenden und aufschlussreichen Artikel von Philip J. Davis [5].

\section{V. Thébaults Aufgabe}

Widmen wir uns nun einigen der zahlreichen Aufgaben V. Thébaults. Zu den populärsten Vertretern zählt zweifelsfrei die Aufgabe zu den drei Kreisen mit kollinearen Mittelpunkten. Erschienen ist diese Aufgabe in der Rubrik „Problems and Solutions“ des Journals American Mathematical Monthly im Jahre 1938 [22].

So lautet die genaue Formulierung:

Through the vertex $A$ of a triangle $A B C$ a straight line $A M$ is drawn cutting the side $B C$ in $M$. Let $2 \theta$ be the angle $A M C ; O$ and $I$ the centers of the circumscribed circle $(O)$ and the inscribed circle $(I)$ of $A B C$. The circles $\left(I_{1}\right)$ and $\left(I_{2}\right)$ with centers $I_{1}$ and $I_{2}$ and radii $\rho_{1}$ and $\rho_{2}$ are each tangent to $(O)$ and the first is tangent also to the two sides of the angle $A M C$ while the second is tangent to the two sides of the angle $A M B$. Prove that:

(1) The straight line joining $I_{1}$ and $I_{2}$ passes through $I$.

(2) The point $I$ divides the segment $I_{1} I_{2}$ in the ratio $\tan ^{2} \theta: 1$; and $\rho_{1}+\rho_{2}=r^{2} \cdot \sec ^{2} \theta$, where $r$ is the radius of $(I)$.

Die letzte Aussage über $\rho_{1}$ und $\rho_{2}$ war leider falsch (Fig. 1).

Erste metrische Lösungen dieser Aufgabe tauchten in den Niederlanden im Jahre 1973 auf, ohne jedoch allzu große Beachtung zu erlangen. Aus diesem Grund veröffentlichte der American Mathematical Monthly im Jahre 1983 eine gekürzte Fassung der, wie es damals schien, ersten metrischen Lösung des Engländers K.B. Taylor. Sie erstreckte sich auf 24 Seiten und beinhaltete mehrere Formeln [19]. 1986 erschien in der Zeitschrift Elemente 


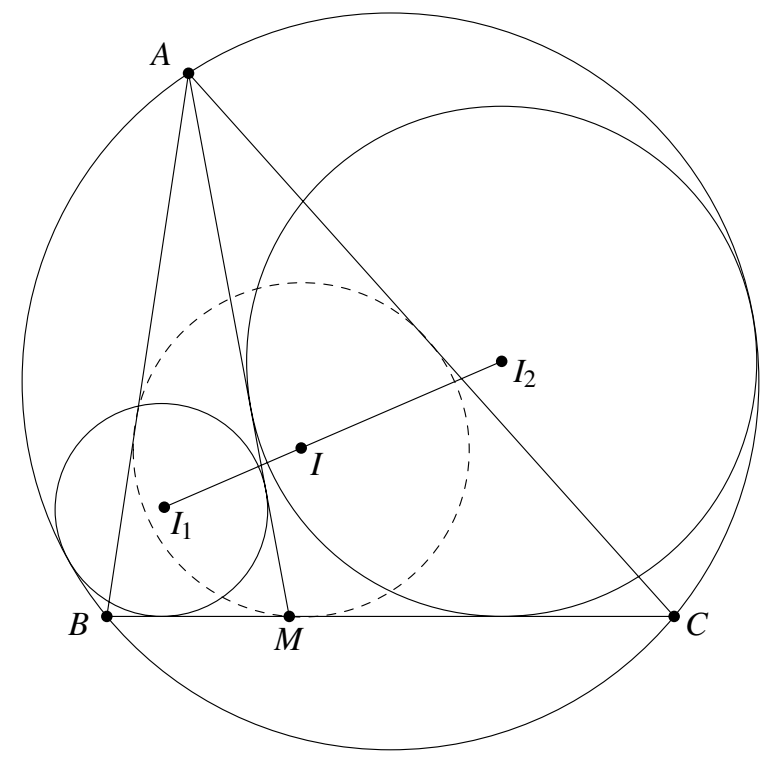

Fig. 1

der Mathematik eine um einiges kürzere metrische Lösung von G. Turnwald [24], und schließlich publizierte der Schweizer Lehrer R. Stärk [18] im selben Jahr und in derselben Zeitschrift die erste synthetische Lösung.

Seitdem erschienen mehrere elementare synthetische Lösungen, zu deren erfolgreichsten die von V. Protassov [8] und zu den letzten die Lösung von Dimitrios Kodokostas [11] zählen. Jean-Louis Ayme, in dessen Artikel eine ausführliche Geschichte und die synthetische Lösung der Aufgabe von Thébault zu finden ist [1], fand heraus, dass jene Aufgabe ein Nachfolger des im Jahre 1905 von dem Mathematiklehrer Sawayama aus Tokio bewiesenen Satzes ist [15].

\section{Variationen des Themas der Thébault-Aufgaben}

In Wirklichkeit ist der Satz von Thébault über die drei Kreise mit den kollinearen Mittelpunkten gleichermassen auf die Ankreise anwendbar. Genauer, wenn $D$ ein beliebiger Punkt auf der Geraden $A C$ des Dreiecks $A B C$ ist; $I_{1}$ Mittelpunkt des Kreises, der die Geraden $A D, B D$ und den Umkreis des Dreiecks $A B C$ von außen berührt; $I_{2}$ Mittelpunkt des Kreises, der die Geraden $C D, B D$ und den Umkreis des Dreiecks $A B C$ von außen berührt, dann verläuft die Gerade $I_{1} I_{2}$ durch den Mittelpunkt $I_{b}$ des Ankreises des Dreiecks $A B C$, und berührt dabei die Seite $A C$.

Führen wir zwei weitere Aufgaben, die nicht von Thébault stammen, an, die eng mit der Aufgabe 3887 verbunden sind.

1. Ein Kreis berührt die verlängerten Seiten $C A$ und $C B$ des Dreiecks $A B C$, berührt ebenfalls die Seite $A B$ im Punkt $P$. Beweise, dass der Radius des Kreises, der die 


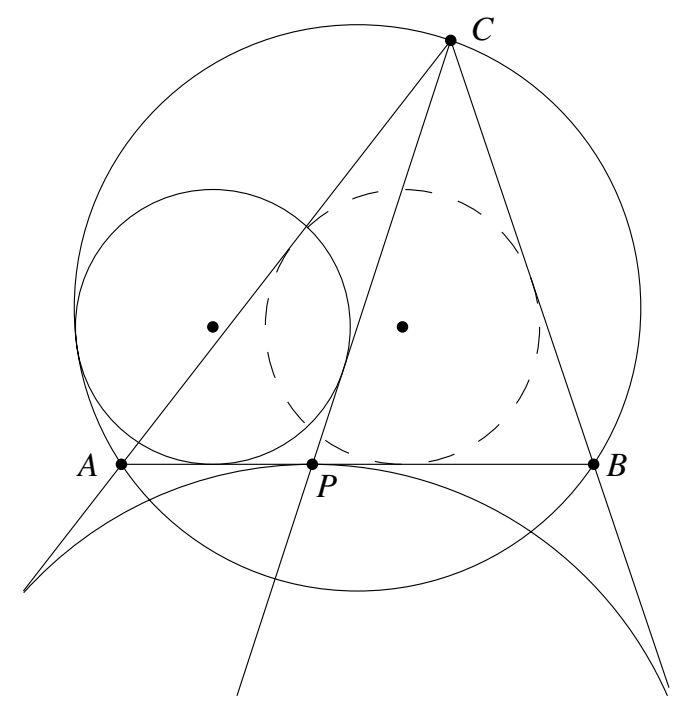

Fig. 2

Strecken $A P, C P$ und den Umkreis des Dreiecks $A B C$ berührt, dem Inkreisradius des Dreiecks gleich ist [16] (Fig. 2).

2. Gegeben sei ein Dreieck $A B C$. Sei $K$ der Berührungspunkt des Inkreises des Dreiecks $A B C$ mit der Seite $B C$. Betrachten wir zwei Kreise, die die Gerade $B C$, den Strahl $A K$ und den Umkreis des Dreiecks berühren. $\mathrm{Zu}$ beweisen ist, dass deren Radius genauso groß ist, wie der Radius des Ankreises des Dreiecks $A B C$, der die Seite $B C$ und die Verlängerungen der Seiten $A B$ und $A C$ berührt [14] (Fig. 3).

Und noch eine Aufgabe Thébaults. Es handelt sich um die Aufgabe 4328, die im Jahre 1949 den Weg in den American Mathematical Monthly fand [22]: „Given a triangle $A B C$ whose altitudes are $A A^{\prime}, B B^{\prime}, C C^{\prime}$. Prove that the Euler lines of the triangles $A B^{\prime} C^{\prime}$, $B C^{\prime} A^{\prime}, C A^{\prime} B^{\prime}$ are concurrent on the nine-point circle at a point $P$ which is such that one of the distances $P A^{\prime}, P B^{\prime}, P C^{\prime}$ equals the sum of the other two" (Fig. 4).

E.D. Kulanin entwickelte in seinen Artikeln die elementare synthetische Lösung der Aufgabe 4328 ([12], [13]). Der Punkt $P$, in dem sich die Euler-Geraden der Dreiecke $A B^{\prime} C^{\prime}$, $B C^{\prime} A^{\prime}, C A^{\prime} B^{\prime}$ schneiden, ist zugleich der Mittelpunkt einer gleichseitigen Hyperbel, die eine isogonale Bildform der Euler-Geraden des Dreiecks $A B C$ darstellt. Die Hyperbel wird Jerabek-Hyperbel genannt. Den Schnittpunkt der Euler-Geraden der Dreiecke $A B^{\prime} C^{\prime}$, $B C^{\prime} A^{\prime}, C A^{\prime} B^{\prime}$ bezeichnen im weiteren als Thébault-Punkt $T$.

In den Artikeln [12] und [13] von E.D. Kulanin wird der folgende Satz bewiesen: Gegeben sei der Schnittpunkt $H$ der Höhen eines spitzwinkligen Dreiecks $A B C ; \mathrm{H}_{1}, \mathrm{H}_{2}, \mathrm{H}_{3}$ seien die Fußpunkte der Höhen; $H_{1}^{\prime} H_{2}^{\prime} H_{3}^{\prime}$ bezeichne das Dreieck, dessen Mitteldreieck gleich dem Dreieck $\mathrm{H}_{1} \mathrm{H}_{2} \mathrm{H}_{3}$ ist. Dann sind die Thébault-Punkte T, $T_{a}, T_{b}, T_{c}$ der Dreiecke ABC, $B H C, C H A, A H B$ identisch mit den Feuerbach-Punkten [11] des Dreiecks $H_{1}^{\prime} H_{2}^{\prime} H_{3}^{\prime}$, 


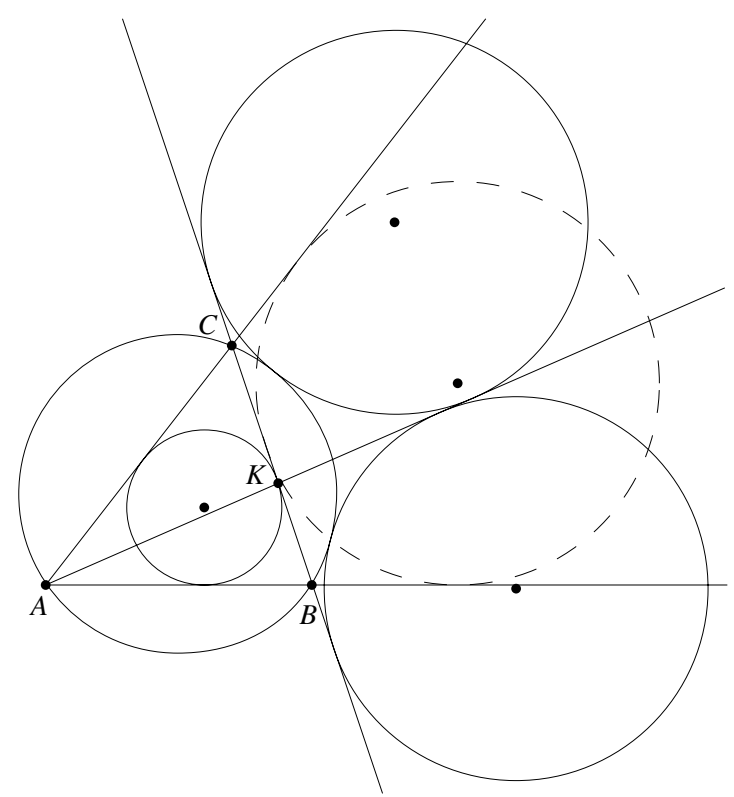

Fig. 3

wobei der Punkt $T$ mit dem inneren und die Punkte $T_{a}, T_{b}, T_{c}$ mit den äußeren FeuerbachPunkten des Dreiecks $\mathrm{H}_{1}^{\prime} \mathrm{H}_{2}^{\prime} \mathrm{H}_{3}^{\prime}$ identisch sind.

Somit besitzen die Feuerbach-Punkte eines beliebigen Dreiecks auch die Eigenschaften der Thébault-Punkte, und daraus folgt: Der größte Abstand von einem beliebigen Feuerbach-Punkt eines Dreiecks zum Mittelpunkt der Seite des Dreiecks entspricht der Summe der Abstände von dem gleichen Feuerbach-Punkt zu den zwei Mittelpunkten der übrigen Dreiecksseiten.

Schließlich die letzte Aufgabe 4432, eng verbunden mit der vorhergehenden [23] (Fig. 5): „With the midpoints of the sides of a triangle as center circles are described passing through the feet of the corresponding altitudes. Show that the circle orthogonal to these three circles is tangent to the nine-point circle of the given triangle at a point such that its distance from the foot of one altitude is equal to the sum of the distances from the feet of the other two altitudes".

Im Artikel [13] wird gezeigt, dass auf dem Umkreis eines beliebigen Dreiecks genau vier Punkte existieren, die folgende Eigenschaften besitzen: Der größte Abstand von einem der vier Punkte zu einem der Eckpunkte des Dreiecks ist gleich der Summe der Abstände von diesem Punkt zu den anderen zwei Eckpunkten des Dreiecks. Wenn man durch die Eckpunkte des Dreiecks Geraden zieht, die zu seinen gegenüberliegenden Seiten parallel sind, dann liegen diese Punkte auf dem mit Feuerbach-Punkten gezeichneten Dreieck. Wenn man jedoch durch die Eckpunkte des Dreiecks Geraden zieht, die senkrecht zu aus diesen Eckpunkten hervorgehenden Winkelhalbierenden sind, dann liegen diese Punkte 


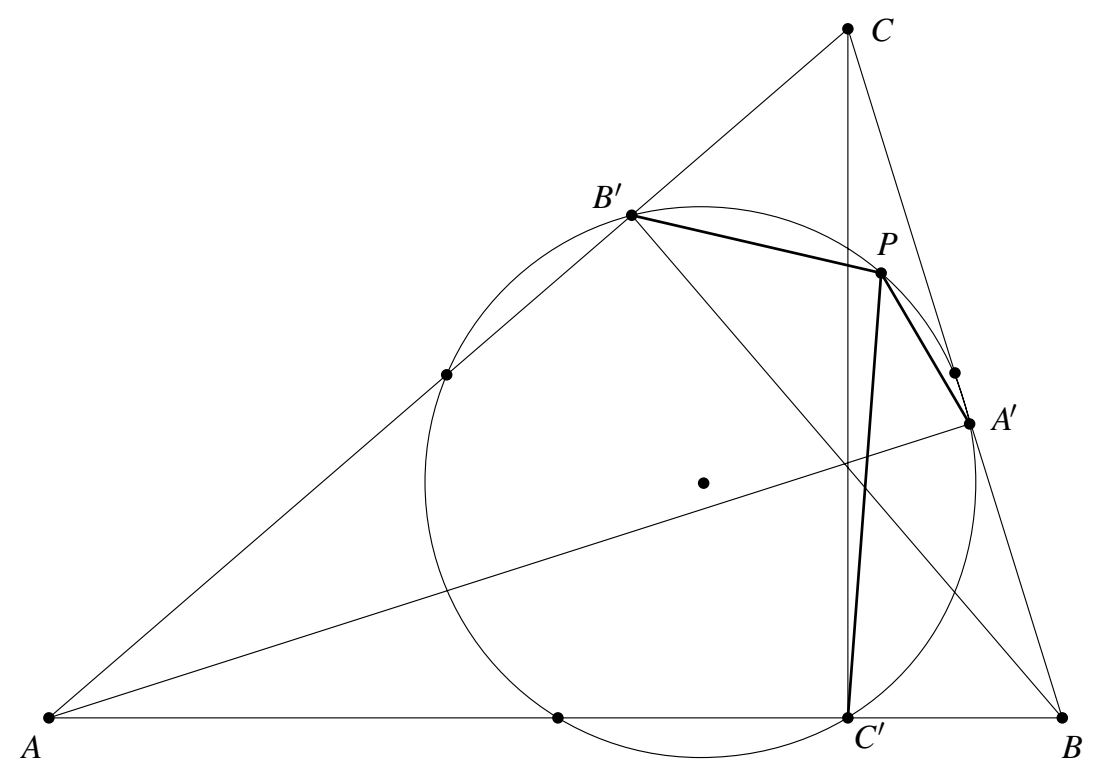

Fig. 4

auf dem mit Thébault-Punkten gezeichneten Dreieck. Aus alledem wird ersichtlich, dass in den Aufgaben 4328 und 4432 die Rede von ein und demselben Punkt ist, den wir in diesem Artikel als Thébault-Punkt bezeichnet haben.

Nennen wir Kreise, deren Mittelpunkte in der Mitte der Dreiecksseiten liegen und die durch die Fußpunkte der Höhen ebenjener Seiten verlaufen, orthomedial. Es sei $H$ der Schnittpunkt der Höhen eines spitzwinkligen Dreiecks $A B C$; weiter seien $S, S_{a}, S_{b}, S_{c}$ die Potenzpunkte orthomedialer Kreise der Dreiecke $A B C, B H C, C H A, A H B$. Kreise mit Mittelpunkten in solchen Punkten, die durch die Thébault-Punkte $T, T_{a}, T_{b}, T_{c}$ entsprechend der Dreiecke $A B C, B H C, C H A, A H B$ verlaufen, nennen wir Thébault-Kreise; sei $E$ gemeinsamer Kreis von neun Punkten der Dreiecke $A B C, B H C, C H A, A H B$. Es ist klar, dass die Vierecke $S S_{a} S_{b} S_{c}$ und $T T_{a} T_{b} T_{c}$ perspektivische Vierecke sind, deren Mittelpunkte mit dem Zentrum $E$ der Perspektive zusammenfallen. Damit ziehen wir aus der Lösung der Aufgabe 4432 folgende Schlussfolgerung: Die Gauß-Geraden des Vierecks, bestehend aus den radikalen Achsen der Kreise der neun Punkte und aus den ThébaultKreisen der Dreiecke $A B C, B H C, C H A, A H B$, also den radikalen Achsen der Kreise $E$ und $S, E$ und $S_{a}, E$ und $S_{b}, E$ und $S_{c}$, ist mit der Euler-Geraden des orthozentrischen Dreiecks der Dreiecke $A B C, B H C, C H A, A H B$ identisch.

Führen wir noch einige weitere interessante Fakten auf, die sich auf die besprochene Konfiguration beziehen.

1. Radikale Achsen der Kreise $S_{a}, S_{b}, S_{c}$ sind mit den Höhen des Dreiecks $A B C$ identisch; die radikalen Achsen der Kreise $S$ und $S_{a}, S$ und $S_{b}, S$ und $S_{c}$ sind mit den Seiten 


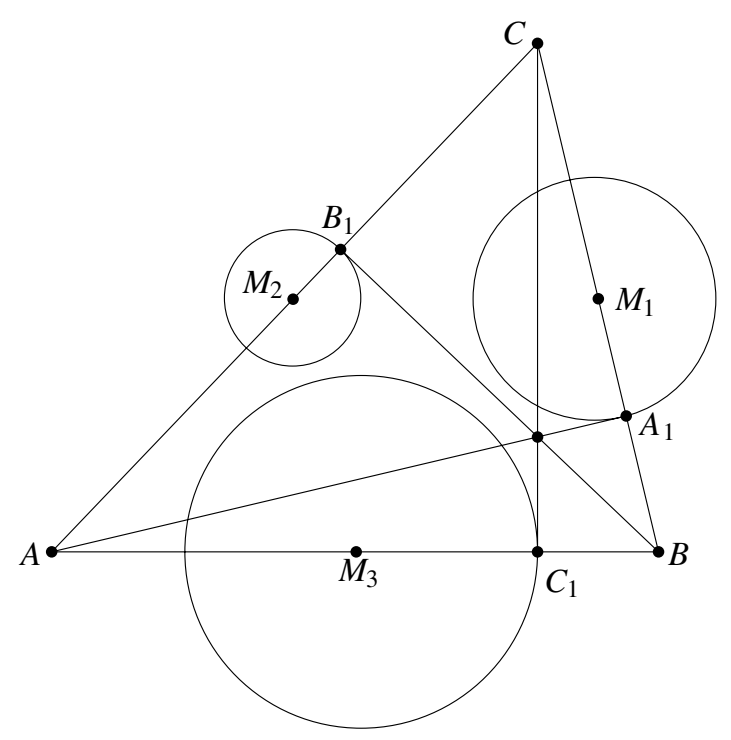

Fig. 5

des Dreiecks $A B C$ identisch, so dass $A, B, C, H$ die radikalen Mittelpunkte der entsprechenden dreifachen Kreise sind.

Interessant ist der Vergleich zwischen der letztgenannten Ausführung mit dem analogen Resultat [7], das sich auf die orthozentroidalen Kreise der Dreiecke $A B C, B H C, C H A$, $A H B$ bezieht. Dieses Resultat fanden wir in der Aufstellung des berühmten Satzes von J. Griffith ([6], [9]): Radikale Achsen der orthozentroidalen Kreise der Dreiecke B HC, $C H A, A H B$ sind mit den Höhen des Dreiecks $A B C$ identisch; radikale Achsen der orthozentroidalen Kreise der Dreiecke $A B C$ und $B H C, A B C$ und $C H A, A B C$ und $A H B$ sind mit den Seiten des Dreiecks $A B C$ identisch, so dass $A, B, C, H$ die radikalen Mittelpunkte der entsprechenden dreifachen Umkreise sind.

2. Das Dreieck $S_{a} S_{b} S_{c}$ ist dem Dreieck $A B C$ ähnlich. Dabei ist das Ähnlichkeitszentrum identisch mit dem Schwerpunkt des orthozentrischen Dreiecks des Dreiecks $A B C$; der Ähnlichkeitsfaktor beträgt -2 .

Die Strecken, welche die Eckpunkte des Dreiecks mit den Berührungspunkten der Ankreise der gegenüberliegenden Seiten verbinden, schneiden sich im Punkt $N$, der NagelPunkt (Fig. 6) genannt wird. Tatsächlich existieren drei weitere Nagel-Punkte $N_{a}, N_{b}$, $N_{c}$. Der Punkt $N_{c}$ ist mit dem Schnittpunkt dreier Geraden identisch: die erste Gerade verläuft durch den Eckpunkt $A$ und den Berührungspunkt des Ankreises $I_{b}$ der Seite $A C$; die zweite Gerade verläuft durch den Eckpunkt $B$ und den Berührungspunkt des Ankreises $I_{a}$ der Seite $B C$; die dritte Gerade verläuft durch den Eckpunkt $C$ und den Berührungspunkt des Inkreises $I$ der Seite $A B$ (Fig. 7). Die Punkte $N_{a}$ und $N_{b}$ lassen sich analog bestimmen. Dies ist durch den Satz von Ceva leicht zu beweisen. 


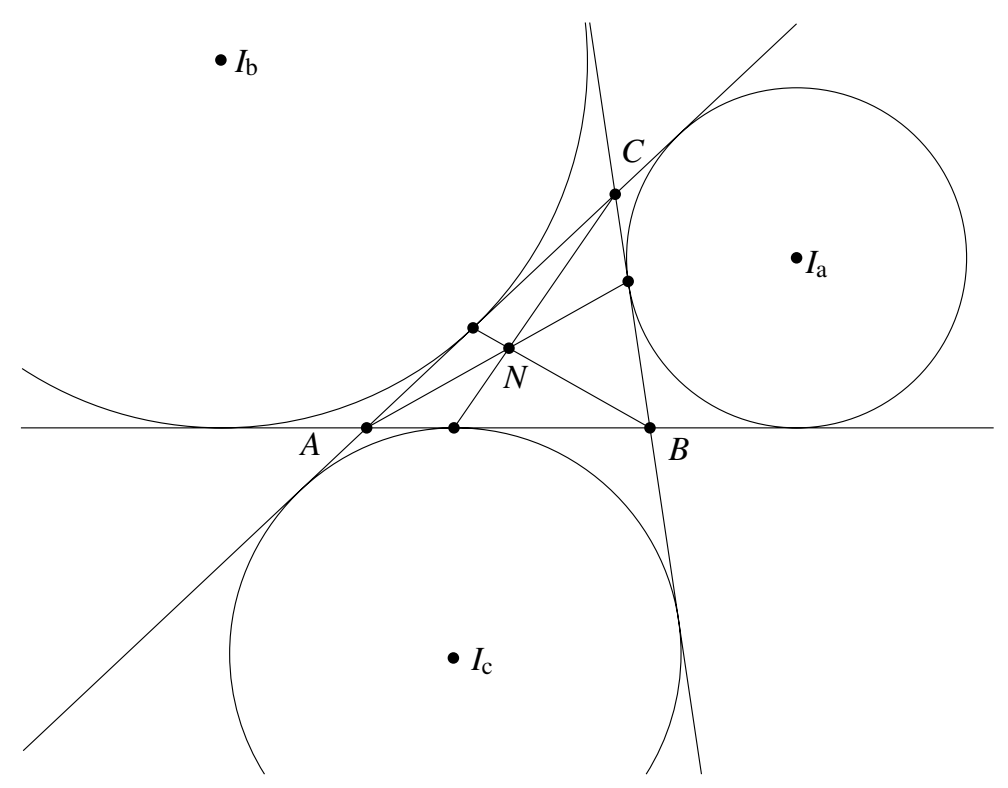

Fig. 6

Die Punkte $N_{a}, N_{b}, N_{c}$ werden wir als äußere Nagel-Punkte bezeichnen. Ohne Beweis führen wir die folgenden drei Sätze an:

Satz 1. Das Viereck, dessen Eckpunkte aus den Nagel-Punkten des Dreiecks ABC bestehen, ist ähnlich dem Viereck, dessen Eckpunkte von den Mittelpunkten des Inkreises bzw. den Ankreisen desselben Dreiecks gebildet werden. Das Ähnlichkeitszentrum ist mit dem Schwerpunkt des orthozentrischen Dreiecks des Dreiecks ABC identisch; der Ähnlichkeitsfaktor beträgt -2 .

Satz 2. Es sei $O$ der Mittelpunkt des Umkreises eines beliebigen Dreiecks; $N, N_{a}, N_{b}, N_{c}$ seien seine Nagel-Punkte. Dann sind vier Punkte des Umkreises dieses Dreiecks so, dass der Abstand eines dieser Punkte zu einem der Eckpunkte des Dreiecks gleich der Summe der Abstände zwischen diesem Punkt und den beiden anderen Eckpunkten des Dreiecks ist, und diese liegen einzeln auf den Geraden $O N, O N_{a}, O N_{b}, O N_{c}$.

Satz 1 erscheint als Folge eines altbekannten Faktes: Abschnitte, die die entsprechenden Nagel-Punkte des gegebenen Dreiecks mit den Mittelpunkten seines In- bzw. seiner Ankreise verbinden, schneiden sich im Schwerpunkt dieses Dreiecks und teilen ihn im Verhältnis $2: 1$, beginnend ab den Nagel-Punkten.

Für den Beweis des Satzes 2 muss man darüber hinaus den Satz von Feuerbach heranziehen und den Umstand beachten, dass am Umkreis eines beliebigen Dreiecks genau vier solcher Punkte existieren, so dass der größte Abstand von einem dieser Punkte zu dem Eckpunkt des Dreiecks gleich der Summe des Abstandes zwischen demselben Punkt zu den zwei anderen Eckpunkten ist. 


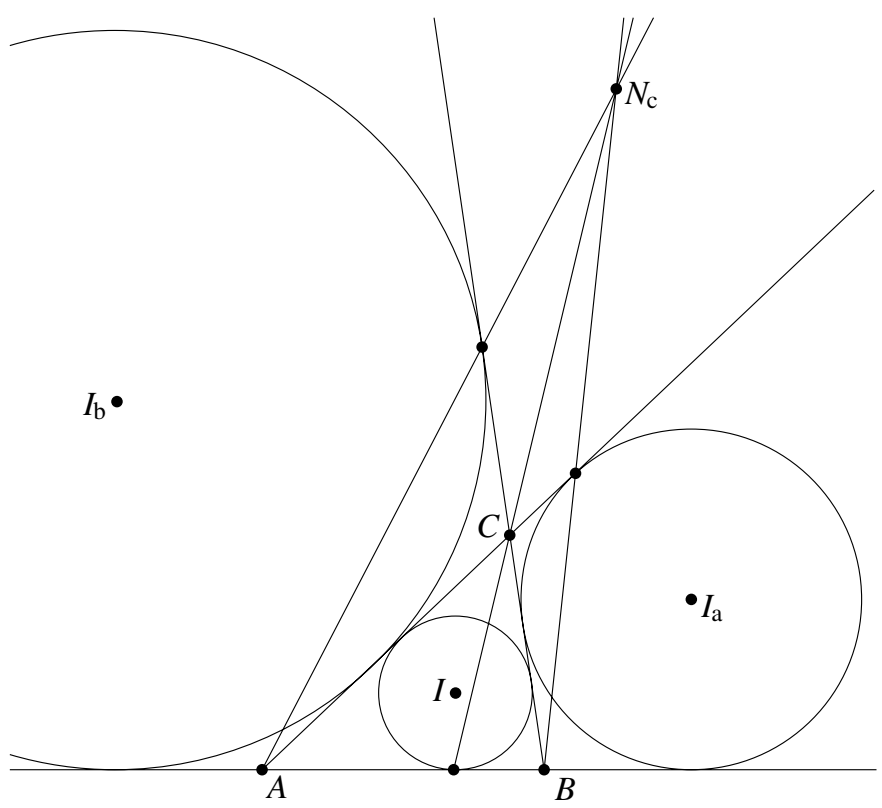

Fig. 7

Satz 3. Die Nagel-Punkte des orthozentrischen Dreiecks $H_{a} H_{b} H_{c}$ eines Dreiecks ABC sind mit den radikalen Mittelpunkten der orthomedialen Umkreise der Dreiecke $A B C$, $B H C, C H A, A H B$, also mit den Punkten $S, S_{a}, S_{b}, S_{c}$, identisch.

Da wir uns entschieden haben, die Umkreise $S, S_{a}, S_{b}, S_{c}$ als Thébault-Kreise zu benennen, fällt die Formulierung des dritten Satzes wie folgt aus:

Satz 3'. Die Mittelpunkte der Thébault-Kreise der Dreiecke ABC, BHC, CHA, AH B sind mit den Nagel-Punkten eines gemeinsamen orthozentrischen Dreiecks all dieser Dreiecke identisch.

Nun wird deutlich, dass die Aussage 2 aus dem Resultat der Aufgabe 4432 und den Sätzen 1-3 hervorgeht.

Führen wir noch eine weitere Variation zum gleichen Thema an:

Gegeben seien $M_{a}, M_{b}, M_{c}$ als Mittelpunkte der Seiten $B C, C A, A B$ des Dreiecks $A B C$; $I, I_{a}, I_{b}, I_{c}$ seien die Mittelpunkte des In- bzw. der Ankreise des Dreiecks $M_{a} M_{b} M_{c}$; $N, N_{a}, N_{b}, N_{c}$ seien die Nagel-Punkte des Dreiecks $M_{a} M_{b} M_{c} ; F, F_{a}, F_{b}, F_{c}$ seien die Feuerbach-Punkte des Dreiecks $A B C$. Dann berühren die Kreise mit den Mittelpunkten in $N, N_{a}, N_{b}, N_{c}$, die durch die Punkte $F, F_{a}, F_{b}, F_{c}$ verlaufen, den Kreis der neun Punkte (Euler) des Dreiecks $A B C$ in den Punkten $F, F_{a}, F_{b}, F_{c}$ und sind orthogonal zu den orthomedialen Kreisen entsprechend der Dreiecke $I_{a} I_{b} I_{c}, I I_{b} I_{c}, I_{a} I I_{c}, I_{a} I_{b} I$. 
Zum Abschluss soll angemerkt werden, dass die Aussage 1 eng mit dem folgenden Fakt verbunden ist: $I, I_{a}, I_{b}, I_{c}$ sollen den In- bzw. die Ankreise des Dreiecks $A B C$ bezeichnen. Dann bilden die radikalen Achsen der Kreise $I_{a}, I_{b}, I_{c}$ ein Dreieck, das dem Dreieck $I_{a} I_{b} I_{c}$ ähnlich ist mit Ähnlichkeitszentrum identisch dem Schwerpunkt des Dreiecks $A B C$; der Ähnlichkeitsfaktor beträgt $-1 / 2$. Hierbei schließen die radikalen Achsen der Kreise $I$ und $I_{a}, I$ und $I_{b}, I$ und $I_{c}$ die Höhen des Dreiecks ein. Des weiteren sind der Höhenschnittpunkt und die Ecken dieses Dreiecks mit den Mittelpunkten des In- bzw. der Ankreise des Dreiecks identisch; als Eckpunkte dienen dabei die Mittelpunkte der Seiten des Ausgangsdreiecks $A B C$.

Aus alledem wird ersichtlich, dass der Thébault-Kreis eines spitzwinkligen Dreiecks identisch ist mit dem Inkreis eines Dreiecks, dessen Seitenmittelpunkte gleichzeitig Höhenfußpunkte des spitzwinkligen Ausgangsdreiecks sind.

Der Thébault-Kreis eines stumpfwinkligen Dreiecks jedoch ist mit einem der Ankreise des Dreiecks identisch, dessen Seitenmittelpunkte gleichzeitig die Höhenfußpunkte des stumpfwinkligen Ausgangsdreiecks sind.

\section{Literatur}

[1] Ayme, J.-L.: Sawayama and Thébault's theorem. Forum Geometricorum 3 (2003), 225-229.

[2] Berkhan, G.; Meyer, W.Fr.: Neuere Dreiecksgeometrie. Vol. III, AB 10 (1914), 1173-1276.

[3] Byrne, W.E.: Victor Thébault - The man. Amer. Math. Monthly 54 (1947), 443-444.

[4] Court, N.A.: Thébault - The geometer. Amer. Math. Monthly 54 (1947), 445-446.

[5] Davis, P.J.: The Rise, Fall and Possible Transfiguration of Triangle Geometry: A Mini-history. Amer. Math. Monthly 102 (1995), 204-214.

[6] Droussent, L.: On a theorem of J. Griffiths. Amer. Math. Monthly 54 (1947), 538-540.

[7] Droussent, L.: On the orthocentroidal circle. Amer. Math. Monthly 57 (1950), 169-171.

[8] Elective course on mathematics, Moscow, "Prosvescheniye" (1991), 341-343 (Russian).

[9] Griffiths, J.: Nouvelles Annales de Mathématiques (1864), 345 und (1865), 322.

[10] Klein, F.: Elementarmathematik vom höheren Standpunkt aus. Bd. 2: Geometrie. In: Grundlehren der mathematischen Wissenschaften, Bd. 15. Teubner Verlag, Leipzig 1909, 443-444.

[11] Kodokostas, D.: A really elementary proof of Thébaults theorem. Worcester Polytechnic Institute, USA. http://users.wpi.edu/ goulet/mme518_ 2004/Project\%232_ 2004/thebth.pdf\#search=\%22Dimitrios $\% 20$ Kodokostas $\% 20 \% 22$

[12] Kulanin, E.D.: On the certain property of Feuerbach points. Supplement Mathematics for paper First September 10 (1997), Moscow (Russian).

[13] Kulanin, E.D.: On the certain properties of Feuerbach and Thébault points. Supplement Mathematics for paper First September 15 (2005), Moscow (Russian).

[14] Mathematical Prosvescheniye, Third series, issue 5, MCCME, Moscow (2001), 218-219 (Russian).

[15] Sawayama, Y.: A new geometrical proposition. Amer. Math. Monthly 12 (1905), 222-224.

[16] IY Soros School Olympiad 1997-1998, Moscow Centers for Continuous Mathematical Education, 102 , 112-113 (Russian)

[17] Starke, E.P.: Thébault - The number theorist. Amer. Math. Monthly 53 (1947), 445.

[18] Stärk, R.: Eine weitere Lösung der Thébaultschen Aufgabe. Elem. Math. 41 (1989), 130-133.

[19] Taylor, K.B.: Solution of Problem 3887. Amer. Math. Monthly 90 (1983), 482-487. 
[20] Tchebychef, P.L.: Complete works. Edition of USSR Academy of Sciences, Vol. Y, 1951, 446 (Russian).

[21] Thébault, V.: Problem 3887. Three circles with collinear centers. Amer. Math. Monthly 45 (1938), 482483.

[22] Thébault, V.: Problem 4328. Amer. Math. Monthly 56 (1949), 39.

[23] Thébault, V.: Problem 4432. Amer. Math. Monthly 58 (1951), 195.

[24] Turnwald, G.: Über eine Vermutung von Thébault. Elem. Math. 41 (1986), 11-13.

Evgeniy D. Kulanin

Moskauer städtische psychologisch-pädagogische Universität

Moskau, Russland

e-mail: lucas03@mail.ru

Oleh Faynshteyn

Leipzig, Deutschland

e-mail: ofaynshteyn@gmx.de 